\title{
Virtual Histology Intravascular Ultrasound Compared With Optical Coherence Tomography for Identification of Thin-Cap Fibroatheroma
}

\author{
Takashi Kubo, ${ }^{1,2}$ MD, Nobuo Nakamura, ${ }^{1}$ MD, Yoshiki Matsuo, ${ }^{1,2}$ MD, Yasushi Oкumoto, ${ }^{2}$ MD, \\ XiaoFan Wu, ${ }^{3} \mathrm{MD}$, So-Yeon Chor,${ }^{4} \mathrm{MD}$, Kenichi KomukaI, ${ }_{1}^{1} \mathrm{MD}$, Takashi Tanimoto, ${ }^{1} \mathrm{MD}$, \\ Yasushi Ino, ${ }^{1}$ MD, Hironori Kitabata, ${ }^{1}$ MD, Keizo Kimura, ${ }_{1}^{1}$ MD, Masato Mizukoshi, ${ }^{1}$ MD, \\ Toshio Imanishi, ${ }^{1} \mathrm{MD}$, Hideharu AKagi, ${ }^{2} \mathrm{MD}$, Tadao Yamamoto, ${ }^{2} \mathrm{MD}$, and Takashi AKASAKA, ${ }^{1} \mathrm{MD}$

\section{SUMMARY} \\ Virtual histology intravascular ultrasound (VH-IVUS) allows detailed assessment of plaque composition in the \\ clinical setting. Optical coherence tomography (OCT) has been developed as a high-resolution imaging method, which \\ might be a promising technique to identify thin-cap fibroatheroma (TCFA) in vivo. The purpose of the present study was \\ to evaluate the diagnostic accuracy of VH-IVUS to identify TCFA as determined by OCT. \\ We examined 96 target lesions in patients with stable angina pectoris by using VH-IVUS and OCT. VH-IVUS de- \\ rived TCFA was defined as a focal necrotic core-rich lesion without evident overlying fibrous tissue. OCT derived TCFA \\ was defined as a plaque with a fibrous cap of $<65 \mu \mathrm{m}$. VH-IVUS correctly identified 16 TCFA and 67 non-TCFA but \\ misclassified 2 TCFA and 11 non-TCFA as determined by OCT. The sensitivity, specificity, and positive and negative \\ predictive values of VH-IVUS to identify TCFA as determined by OCT were $89 \%, 86 \%, 59 \%$, and $97 \%$, respectively. \\ VH-IVUS showed an acceptable sensitivity and specificity to identify TCFA as determined by OCT. Although the \\ positive predictive value was low reflecting a high number of false positives, the negative predictive value was notably \\ high. Our results suggest a potential role for VH-IVUS to exclude high risk lesions for future coronary events. (Int \\ Heart J 2011; 52: 175-179)
}

Key words: Intravascular ultrasound, Virtual histology, Optical coherence tomography, Coronary artery disease

$\mathrm{P}$ aque rupture is the most common cause of coronary thrombosis which leads to acute coronary syndrome and sudden coronary death. Several autopsy studies have proposed that thin-cap fibroatheroma (TCFA) with a cap thickness of $<65 \mu \mathrm{m}$ is a precursor lesion for plaque rupture. ${ }^{1,2)}$ It has been recognized that identification of TCFA in living patients might help reduce the incidence of acute coronary events. Intravascular optical coherence tomography (OCT) has been developed as a high-resolution imaging method. ${ }^{3)}$ It has a resolution of 10 to $20 \mu \mathrm{m}$ and allows us to identify a thin fibrous cap precisely in vivo. However, OCT is a specialized research tool which requires complex procedures for image acquisition.

Intravascular ultrasound (IVUS) is the gold standard for evaluation of coronary plaque morphology in routine clinical practice. Recently, spectral analysis of IVUS radiofrequency data (Virtual Histology IVUS [VH-IVUS]) has demonstrated potential to differentiate 4 components of atherosclerotic plaques (ie, fibrous, fibro-fatty, dense calcium, and necrotic core). ${ }^{4)}$ Because the axial resolution of IVUS is between 100 to $150 \mu \mathrm{m}$, several VH-IVUS studies have proposed that the ab- sence of visible fibrous tissue overlying a necrotic core indicated a cap thickness of less than 100 to $150 \mu \mathrm{m}$ and used the absence of such tissue to identify TCFA. ${ }^{5)}$ In the present study, we evaluated the diagnostic accuracy of VH-IVUS to identify TCFA compared with OCT.

\section{Methods}

Study population: Between November 2005 and October 2010, 96 patients who presented with stable angina pectoris and had de novo lesions in native coronary arteries were studied by using VH-IVUS and OCT at Wakayama Medical University, Wakayama, Japan and Social Insurance Kinan Hospital, Wakayama, Japan. Stable angina pectoris was defined as chest pain related to effort without change in the frequency, duration, or intensity of symptoms. The target lesion was identified by the combination of exercise electrocardiographic findings, scintigraphic reversible defects, and angiographic lesion morphology. In patients with multiple coronary lesions, the lesion with most severe diameter stenosis and most complex le-

From the ${ }^{1}$ Department of Cardiovascular Medicine, Wakayama Medical University, ${ }^{2}$ Department of Cardiovascular Medicine, Social Insurance Kinan Hospital, Wakayama, Japan, ${ }^{3}$ Department of Cardiology, Beijing Anzhen Hospital, Capital Medical University, Beijing, China, and ${ }^{4}$ Department of Cardiology, Ajou University School of Medicine, Suwon, Korea.

Address for correspondence: Takashi Kubo, MD, Department of Cardiovascular Medicine, Social Insurance Kinan Hospital, 46-70 Shinjo-cho, Tanabe City, Wakayama 646-8588, Japan.

Received for publication December 14, 2010

Revised and accepted February 24, 2011. 
sion morphology on the coronary angiography was selected as the target lesion. Patients with a left main coronary artery lesion, chronic total occlusions, extremely tortuous vessels with heavy calcification, or renal insufficiency with serum creatinine $>2.0 \mathrm{mg} / \mathrm{dL}$ were excluded. The ethics committee at each participating institution approved the protocol, and written informed consent was obtained from all patients.

VH-IVUS imaging and analysis: VH-IVUS examination was performed before any intervention and after the intracoronary administration of nitroglycerin $(0.2 \mathrm{mg})$. A phased-array, 20 MHz, 3.2 Fr IVUS catheter (Eagle Eye, Volcano Therapeutics, Inc., Rancho Cordova, CA, USA) was placed distal (> $10 \mathrm{~mm}$ ) to the target lesion and pulled back to the aorto-ostial junction using a motorized catheter pullback system set at $0.5 \mathrm{~mm} / \mathrm{sec}-$ ond. During pullback, grayscale IVUS was recorded, raw radiofrequency data were captured at the top of the R-wave, and reconstruction of the color-coded map by a VH-IVUS data recorder (Volcano Therapeutics, Inc.) was performed. Using custom-built software, grayscale and VH-IVUS images were analyzed by 2 independent observers (K.K. and O.Y.) who were blinded to the OCT findings. When there was discordance between the observers, a consensus reading was obtained. Grayscale IVUS measurements of lumen, external elastic membrane (EEM), and plaque and media (P\&M: defined as EEM minus lumen) cross-sectional areas and plaque burden (defined as P\&M divided by EEM) were performed for every recorded frame. ${ }^{6)}$ VH-IVUS analysis was performed across the minimum luminal area site. The 4 VH-IVUS plaque components (fibrous, fibro-fatty, dense calcium, and necrotic core) were expressed as percentages (relative amounts) of plaque area. Using a previously reported definition, ${ }^{5)} \mathrm{VH}-\mathrm{IVUS}$ derived TCFA (VH-TCFA) was defined as a lesion meeting the following two criteria in at least 3 consecutive frames: (1) focal necrotic core-

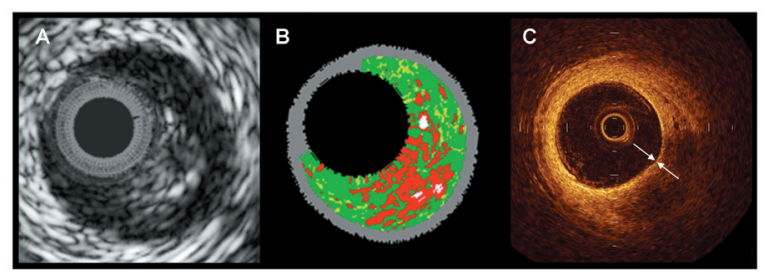

Figure 1. TCFA as determined by VH-IVUS and OCT. (A) GrayscaleIVUS shows eccentric coronary plaque. (B) VH-IVUS demonstrates absence of visible fibrous tissue overlying a necrotic core. (C) OCT visualizes thin fibrous cap of $50 \mu \mathrm{m}$ thickness (arrows).

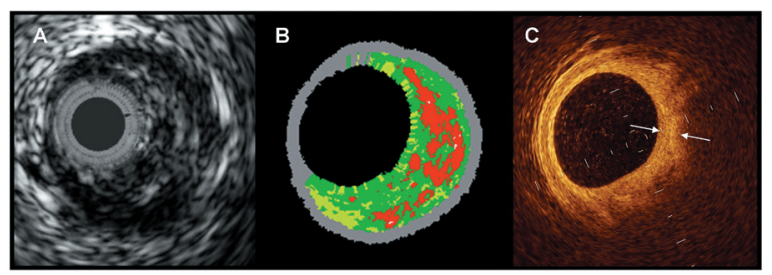

Figure 2. Non-TCFA as determined by VH-IVUS and OCT. (A) Grayscale-IVUS shows eccentric coronary plaque. (B) VH-IVUS demonstrates presence of visible fibrous tissue overlying a necrotic core. (C) OCT visualizes thick fibrous cap of $420 \mu \mathrm{m}$ thickness (arrows). rich lesions (necrotic core $\geq 10 \%$ ) without evident overlying fibrous tissue and (2) plaque burden $\geq 40 \%$. Representative images are shown in Figures 1 and 2.

OCT imaging and analysis: After completion of IVUS, the OCT examination was performed using the M2 OCT imaging system (LightLab Imaging, Inc, Westford, MA, USA). We used a continuous-flushing (nonocclusive) method for OCT image acquisition. ${ }^{7)}$ A 0.014-inch wire-type imaging catheter was positioned distal to the target lesion. To remove blood cells from the field of view, a mixture of commercially available dextran 40 and lactated Ringer solution was infused from the guiding catheter at 2.5 to $4.5 \mathrm{~mL} / \mathrm{second}$ with an injector pump. The target lesion was imaged with a motorized pullback device travelling at $1 \mathrm{~mm} / \mathrm{second}$. Continuous OCT images were stored digitally for subsequent analysis. The OCT analysis was performed by 2 independent observers (T.T. and M.Y.) who were blinded to the VH-IVUS findings. When there was discordance between the observers, a consensus reading was obtained. The distances from at least 2 landmarks, such as side branches and/or calcifications, were used as references in order to obtain corresponding images of IVUS and OCT. Using the validated criteria for plaque characterization, ${ }^{3)}$ the minimum fibrous cap thickness was measured at the minimum luminal area site. OCT derived TCFA (OCT-TCFA) was defined as a plaque with a fibrous cap of $<65 \mu \mathrm{m}$ according to the previous reports. ${ }^{8,9)}$

Quantitative coronary angiographic analysis: Quantitative coronary angiography analysis was performed using the Cardiovascular Measurement System (CMSMEDIS Medical Imaging System, Leiden, The Netherlands). Angiographic lesion morphology was classified according to the ACC/AHA classification. ${ }^{10)}$ The percentage of diameter stenosis was measured in the view that was the most severe and not foreshortened.

Statistical analysis: Statistical analysis was performed using Statview 5.0.1 (SAS Institute, Cary, NC, USA). Categorical variables are presented as counts and percentages. Continuous variables are presented as the mean \pm standard deviation. Intraobserver and interobserver variabilities for VH-TCFA and OCT-TCFA were measured using the $\kappa$ test of concordance. Accuracy (percentage of lesions correctly classified), sensitivity, specificity, and positive and negative predictive values of VH-IVUS for identifying TCFA were calculated on a per-lesion basis.

\section{RESULTS}

The clinical and angiographic characteristics are shown in Table I. Seventy-two patients were male and the mean patient age was 69 years. The most frequent target vessel was the left anterior descending artery (50\%), and the majority of lesions were classified as type B (64\%) according to the ACC/ AHA lesion classification.

The IVUS and OCT findings are shown in Table II. The VH-IVUS images at the minimum luminal area site demonstrated that the percentage of necrotic core area was $20 \pm 7 \%$ and that VH-TCFA was observed in $29(30 \%)$ lesions. Intraobserver and interobserver variabilities for VH-TCFA yielded acceptable concordance ( $\kappa=0.84$ and $\kappa=0.80$, respectively). In the corresponding OCT images, the minimum fibrous cap thickness was $251 \pm 196 \mu \mathrm{m}$, and OCT-TCFA was identified in 
Table I. Clinical and Angiographic Characteristics

\begin{tabular}{lc}
\hline Age, years & $69 \pm 9$ \\
Male & $72(75)$ \\
Hypertension & $69(72)$ \\
Diabetes mellitus & $25(26)$ \\
Hypercholesterolemia & $61(66)$ \\
Current smoker & $26(27)$ \\
Target vessel & \\
$\quad$ LAD & $48(50)$ \\
LCX & $15(16)$ \\
$\quad$ RCA & $33(34)$ \\
ACC/AHA lesion classification & \\
$\quad$ Type A & $31(32)$ \\
Type B1 & $36(38)$ \\
Type B2 & $25(26)$ \\
Type C & $4(4)$ \\
Percent diameter stenosis, $\%$ & $72 \pm 5$ \\
\hline
\end{tabular}

Values are given as $n(\%)$ or mean \pm standard deviation. LAD indicates left anterior descending coronary artery; LCX, left circumflex coronary artery; and RCA, right coronary artery.

Table II. IVUS and OCT Findings at Minimum Luminal Area Site

\begin{tabular}{lc}
\hline Grayscale-IVUS findings & \\
Luminal area, mm ${ }^{2}$ & $3.1 \pm 0.6$ \\
EEM area, $\mathrm{mm}^{2}$ & $14.4 \pm 3.1$ \\
P\&M area, mm ${ }^{2}$ & $11.4 \pm 3.0$ \\
Plaque burden, $\%$ & $78 \pm 5$ \\
VH-IVUS findings & \\
Fibrous, $\%$ & $59 \pm 10$ \\
Fibro-fatty, $\%$ & $13 \pm 7$ \\
Dense calcium, $\%$ & $8 \pm 7$ \\
Necrotic core, $\%$ & $20 \pm 7$ \\
VH-TCFA & $29(30 \%)$ \\
OCT findings & \\
Fibrous cap thickness, $\mu \mathrm{m}$ & $251 \pm 196$ \\
OCT-TCFA & $18(19 \%)$
\end{tabular}

Values are given as $n(\%)$ or mean \pm SD when appropriate. IVUS indicates intravascular ultrasound; OCT, optical coherence tomography; EEM, external elastic membrane; $\mathrm{P} \& \mathrm{M}$, plaque and media; $\mathrm{VH}$, virtual histology; and TCFA, thin-cap fibroatheroma.

Table III. Diagnostic Accuracy of VH-IVUS to Detect TCFA as Determined by OCT

\begin{tabular}{lc}
\hline No. of true-positives & 16 \\
No. of true-negatives & 67 \\
No. of false-positives & 11 \\
No. of false-negatives & 2 \\
Sensitivity, \% & 89 \\
Specificity, \% & 86 \\
Positive predictive value, $\%$ & 59 \\
Negative predictive value, $\%$ & 97 \\
Accuracy, $\%$ & 86
\end{tabular}

VH-IVUS indicates virtual histology intravascular ultrasound; TCFA, thin-cap fibroatheroma; and OCT, optical coherence tomography.

$18(19 \%)$ lesions. Intraobserver and interobserver variabilities for OCT-TCFA showed good concordance $(\kappa=0.86$ and $\kappa=$ 0.84 , respectively).

The diagnostic accuracy of VH-IVUS to detect TCFA as determined by OCT is summarized in Table III. VH-IVUS cor-

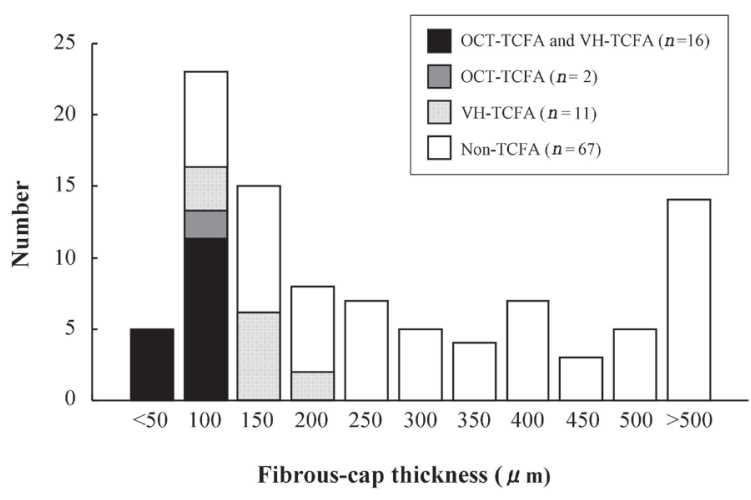

Figure 3. Distribution of fibrous cap thickness measured by OCT. VHIVUS correctly identified 16 TCFA (fibrous cap thickness: $51 \pm 9 \mu \mathrm{m}$ ) and 67 non-TCFA (fibrous cap thickness: $326 \pm 189 \mu \mathrm{m}$ ) but misclassified 2 TCFA (fibrous cap thickness: 57 and $60 \mu \mathrm{m}$, respectively) and 11 non-TCFA (fibrous cap thickness: $117 \pm 27 \mu \mathrm{m}$ ) as determined by OCT.

rectly identified 16 TCFA and 67 non-TCFA, but misclassified 2 TCFA and 11 non-TCFA as determined by OCT. Two lesions with false-negative VH-IVUS imaging contained large superficial calcifications. Eleven lesions with false-positive VH-IVUS imaging had a fibrous cap of $117 \pm 27 \mu \mathrm{m}$ thick (Figure 3). The sensitivity, specificity, and positive and negative predictive values were $89 \%, 86 \%, 59 \%$, and $97 \%$, respectively.

\section{Discussion}

In the present study, VH-IVUS showed an acceptable accuracy to identify TCFA as determined by OCT. VH-IVUS might be a useful modality to assess lesion instability in the clinical setting.

TCFA as defined by pathology: Plaque rupture is the most frequent cause of coronary thrombosis, accounting for $55-60 \%$ of all coronary thrombi. ${ }^{1)}$ It has been postulated that TCFA is the precursor lesion of plaque rupture. TCFA is characterized by a thin fibrous cap of $<65 \mu \mathrm{m}$ heavily infiltrated by macrophages and underlying necrotic core. The thin fibrous cap is distinguished from the earlier fibrous cap lesions by the loss of smooth muscle cells, extracellular matrix, and inflammatory infiltrate. The necrotic core underlying the thin fibrous cap is usually large; hemorrhage and/or calcification is often present; and intraplaque vasa vasorum are abundant. ${ }^{2)}$ The cap thickness of $65 \mu \mathrm{m}$ was chosen as a criterion of instability because in rupture the mean cap thickness was $23 \pm 19 \mu \mathrm{m}$, with $95 \%$ of the caps measuring $<65 \mu \mathrm{m}$. ${ }^{1)}$ In addition, nearly $75 \%$ of TCFA had $>10 \%$ area of the plaque occupied by necrotic core. $^{2)}$ Based on these pathological data, VH-TCFA and OCTTCFA were defined in the pivotal imaging studies.

OCT derived TCFA: The identification of the fibrous cap is important because the thickness of the fibrous cap is a major determinant of plaque vulnerability. A high-resolution imaging technique including OCT may be a promising method with which to identify the thin fibrous cap of atheroma. ${ }^{11-13)}$ In OCT images, the fibrous cap over the necrotic core was identified as a signal-rich layer overlying a signal-poor area. ${ }^{3)}$ Kume, et al 
reported that there was a good correlation of the fibrous cap thickness between OCT and histological examination ( $\mathrm{y}=$ $0.97 \mathrm{x}+28.49 ; r=0.90 ; P<0.001) .{ }^{14)}$ Jang, et al evaluated the OCT appearance of culprit coronary lesions in vivo and demonstrated a higher frequency of OCT-TCFA in acute myocardial infarction compared with unstable or stable angina (72\%, $50 \%$, and $20 \%$, respectively, $P=0.012) .{ }^{8}$ ) However, the current OCT system has several limitations for clinical uses and skill is required for accurate interpretation of the OCT images. In particular, it may be difficult to discriminate between lipid and calcified lesions because both these lesions appear as lowintensity images, usually differentiated in OCT by an unclear border (lipid) or a clear border (calcium). ${ }^{15)}$ The application of a postprocessing color-coding software-based algorithm on analysis of either spectral OCT backscattered data or other optical tissue properties should improve the characterization of atherosclerotic coronary plaques and provide a more objective assessment.

VH-IVUS derived TCFA: VH-IVUS uses advanced radiofrequency analysis of ultrasound signals and allows detailed qualitative and quantitative assessment of plaque composition. Nair, et al reported that the ex vivo accuracies of VH-IVUS were $90.4 \%$ for fibrous tissue, $92.8 \%$ for fibro-fatty, $90.9 \%$ for dense calcium, and $89.5 \%$ for necrotic core. ${ }^{4)}$ Because the axial resolution of VH-IVUS is too coarse $(100-150 \mu \mathrm{m})$ to visualize a thin fibrous cap of $<65 \mu \mathrm{m}$ thick, Rodriguez-Granillo, et al used the absence of visible fibrous tissue overlying a necrotic core to define VH-TCFA. ${ }^{5}$ Several subsequent studies then evaluated the incidence, distribution, and clinical outcomes of VH-TCFA. ${ }^{16-18)}$ However, only a few studies evaluated the diagnostic accuracy of VH-IVUS to identify TCFA. Sawada, et al assessed 126 coronary lesions (culprit and nonculprit lesions) by VH-IVUS and OCT in 56 patients with angina including acute coronary syndrome and demonstrated that the positive ratio of VH-IVUS for detecting definite TCFA, which was defined as a plaque meeting the TCFA criteria in both methods, was $45.9 \% .^{15)}$ Compared to their study, the present study assessed only culprit lesions in patients with stable angina pectoris, and most lesions presented matured fibroatheroma with greater plaque burden and more abundant necrotic core. The lesion selection and the baseline plaque characteristics may have affected the results. Moreover, coronary thrombus could influence the VH-IVUS and OCT assessment of plaque composition. VH-IVUS classifies mural thrombus into fibrous and fibro-fatty tissue, and OCT can not visualize coronary plaque behind the thrombus clearly due to signal attenuation. In the present study, we did not enroll patients with acute coronary syndrome to preclude thrombotic lesions for imaging analysis. In consequence, VH-IVUS showed an acceptable sensitivity and specificity to identify TCFA as determined by OCT. Although the positive predictive value was low reflecting a high number of false positives, the negative predictive value was notably high. Our results suggest a potential role for VHIVUS to exclude high risk lesions for future coronary events.

Study limitations: The present study has several limitations. First, this was an in vivo validation study. A direct comparison between VH-IVUS, OCT, and histopathology is required to confirm our data. Second, extremely tortuous vessels with heavy calcification were excluded because of expected difficulty in advancing the VH-IVUS and OCT catheter. Selection bias is an important limitation of the present study. Third, the limited longitudinal resolution of VH-IVUS imaging, based on electrocardiography-triggered cross-sectional analysis, may have biased the identification of corresponding images of $\mathrm{VH}$ IVUS and OCT. Finally, there were no follow-up data of VHTCFA and OCT-TCFA. Prospective studies are required to investigate the prognostic value of these findings.

Conclusions: In the present study, VH-IVUS correctly identified TCFA as determined by OCT. In particular, the negative predictive value was notably high. Our results suggest a potential role for VH-IVUS to exclude high risk lesions for future coronary events.

\section{REFERENCES}

1. Virmani R, Kolodgie FD, Burke AP, Farb A, Schwartz SM. Lessons from sudden coronary death: a comprehensive morphological classification scheme for atherosclerotic lesions. Arterioscler Thromb Vasc Biol 2000; 20: 1262-75. (Review)

2. Virmani R, Burke AP, Farb A, Kolodgie FD. Pathology of the vulnerable plaque. J Am Coll Cardiol 2006; 47: C13-C8.

3. Yabushita H, Bouma BE, Houser SL, et al. Characterization of human atherosclerosis by optical coherence tomography. Circulation 2002; 106: 1640-5

4. Nair A, Kuban BD, Tuzcu EM, Schoenhagen P, Nissen SE, Vince DG. Coronary plaque classification with intravascular ultrasound radiofrequency data analysis. Circulation 2002; 106: 2200-6.

5. Rodriguez-Granillo GA, García-García HM, Mc Fadden EP, et al. In vivo intravascular ultrasound-derived thin-cap fibroatheroma detection using ultrasound radiofrequency data analysis. J Am Coll Cardiol 2005; 46: 2038-42.

6. Mintz GS, Nissen SE, Anderson WD, et al. American College of Cardiology Clinical Expert Consensus Document on Standards for Acquisition, Measurement and Reporting of Intravascular Ultrasound Studies (IVUS). A report of the American College of Cardiology Task Force on Clinical Expert Consensus Documents. J Am Coll Cardiol 2001; 37: 1478-92. (Review)

7. Kataiwa H, Tanaka A, Kitabata H, Imanishi T, Akasaka T. Safety and usefulness of non-occlusion image acquisition technique for optical coherence tomography. Circ J 2008; 72: 1536-7.

8. Jang IK, Tearney GJ, MacNeill B, et al. In vivo characterization of coronary atherosclerotic plaque by use of optical coherence tomography. Circulation 2005; 111: 1551-5.

9. Kubo T, Imanishi T, Takarada S, et al. Assessment of culprit lesion morphology in acute myocardial infarction: ability of optical coherence tomography compared with intravascular ultrasound and coronary angioscopy. J Am Coll Cardiol 2007; 50: 933-9.

10. Ellis SG, Vandormael MG, Cowley MJ, et al. Coronary morphologic and clinical determinants of procedural outcome with angioplasty for multivessel coronary disease. Implications for patient selection. Multivessel Angioplasty Prognosis Study Group. Circulation 1990; 82: 1193-202.

11. Kume T, Okura H, Yamada R, et al. Frequency and spatial distribution of thin-cap fibroatheroma assessed by 3-vessel intravascular ultrasound and optical coherence tomography: an ex vivo validation and an initial in vivo feasibility study. Circ J 2009; 73 : 1086-91.

12. Tanimoto T, Imanishi T, Tanaka A, et al. Various types of plaque disruption in culprit coronary artery visualized by optical coherence tomography in a patient with unstable angina. Circ J 2009; 73: $187-9$.

13. Kume T, Akasaka T, Kawamoto T, et al. Assessment of coronary intima--media thickness by optical coherence tomography: comparison with intravascular ultrasound. Circ J 2005; 69: 903-7.

14. Kume T, Akasaka T, Kawamoto T, et al. Measurement of the thickness of the fibrous cap by optical coherence tomography. Am Heart J 2006; 152: 755. e1-4.

15. Sawada T, Shite J, Garcia-Garcia HM, et al. Feasibility of com- 
bined use of intravascular ultrasound radiofrequency data analysis and optical coherence tomography for detecting thin-cap fibroatheroma. Eur Heart J. 2008; 29: 1136-46.

16. Hong MK, Mintz GS, Lee CW, et al. Comparison of virtual histology to intravascular ultrasound of culprit coronary lesions in acute coronary syndrome and target coronary lesions in stable angina pectoris. Am J Cardiol 2007; 100: 953-9.

17. Hong MK, Mintz GS, Lee CW, et al. A three-vessel virtual histol- ogy intravascular ultrasound analysis of frequency and distribution of thin-cap fibroatheromas in patients with acute coronary syndrome or stable angina pectoris. Am J Cardiol 2008; 101: 568-72.

18. Kubo T, Maehara A, Mintz GS, et al. The dynamic nature of coronary artery lesion morphology assessed by serial virtual histology intravascular ultrasound tissue characterization. J Am Coll Cardiol 2010; 55: 1590-7. 